\title{
Viral and anatomical approaches for mapping feedback cortico-cortical connections in the visual system
}

\author{
Ami Tsuboi ${ }^{1}$, Toshiaki Suzuki ${ }^{1}$, Masanari Onda ${ }^{1}$, Masahiro Yamaguchi ${ }^{1}$, Nao Morimoto ${ }^{1,2}$, \\ Fumitaka Osakada ${ }^{1,2,3}$
}

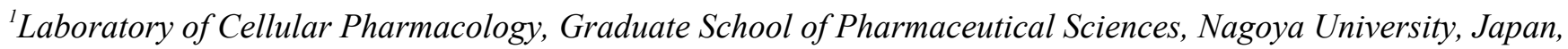
${ }^{2}$ Laboratory of Neural Information Processing, Institute for Advanced Research, Nagoya University, Japan, ${ }^{3}$ PRESTO, Japan Science and Technology Agency, Japan

\section{[Background]}

Visual information begins with the retina and is sent via the thalamus to the visual cortex. The mouse visual cortex consists of a primary visual area (V1) and at least 9 higher-order visual areas (HVAs) which are functionally tuned to specific features of the visual scene. For example, the area AL, one of the HVAs, specializes in processing fast moving, low spatial frequency visual stimuli, while the area PM specializes in processing slow, high temporal frequency stimuli. The functional specialization can arise from the interaction between the V1 and HVAs via feedforward and feedback projections. Thus, revealing how information flows between the V1 and HVAs is pivotal in understanding the mechanisms of visual perception. Little is known, however, about roles of feedback cortico-cortical connections in visual information processing. The aim of our study is to examine how visual properties of V1 neurons providing feedforward inputs to HVAs are shaped by feedback from HVAs. Here we anatomically map feedback connections to HVA-projecting V1 neurons using rabies virus tracing.

[Methods]

Adult C57BL/6 mice stereotaxically received viral injection based on the Allen Mouse Brain Atlas. To label feedback connections between V1 and HVAs, we performed monosynaptically restricted, rabies virus tracing. The brain was harvested and sectioned for anatomical analysis. Visual responses were determined by 2 photon microscopy with genetically encoded $\mathrm{Ca}$ indicators.

[Results]

V1 neurons projecting to one of the HVAs were retrogradely infected with AAV2-retro-CAG-TVA-mCherry and AAV2retro-CAG-H2B-BFP-P2A-oG. EnvA-RV $\triangle$ G-GFP infected the TVA-expressing cells, leading to transsynaptic viral spreading to their presynaptic neurons. The number of virus-infected presynaptic cells in each cortical area was quantified. V1 neurons projecting to a HVA received local inputs from surrounding V1 neurons and feedback inputs from multiple HVAs and other association cortical areas. Interestingly, the proportion of the feedback inputs was biased from a particular HVA among all cortical areas.

\section{[Conclusions]}

Circuit mechanisms are becoming increasingly tractable using the mouse model due to the advantage of genetics and molecular tools. In addition to our anatomical data, viral and genetic approaches combined with in vivo 2-photon imaging and pharmacogenetics will facilitate understanding circuit-level mechanisms underlying cortico-cortical transformations. 